\title{
Aspergillic Acids Produced by Mixed Cultures of Aspergillus flavus and Aspergillus nidulans
}

\author{
By M. J. PERRY,† J. F. MAKINS, $\$$ M. W. ADLARD* AND G. HOLT \\ The Polytechnic of Central London, School of Engineering and Science, \\ 115 New Cavendish Street, London WIM 8JS, UK
}

(Received 1 June 1983; revised 7 October 1983)

\begin{abstract}
A mixed culture of Aspergillus nidulans (GH79) and Aspergillus flavus (CMI 91019B) produced two antibiotics, designated VI and VII, which were not elaborated when either fungus was grown alone. Chemical and spectroscopic analysis of VI, the major component, indicated that this compound was identical to hydroxyaspergillic acid. The minor component, VII, was produced in too low a yield for its identity to be established. However, partial characterization suggests that this antibiotic also belongs to the aspergillic acid group of mycotoxins.
\end{abstract}

\section{INTRODUCTION}

Our laboratory has for some time been engaged in a study of antibiotic biosynthesis by individual strains of Aspergillus nidulans (Cole et al., 1976; Holt et al., 1976; Makins et al., 1980; Perry et al., 1982a,b). However, there is scant information in the literature concerning the use of mixed cultures of aspergilli to produce new metabolites. This is probably due to the difficulty in obtaining stable, mixed microbial systems suitable for subsequent commercial development. However, the progress made in recombinational techniques (Ferenczy, 1979; Hopwood \& Chater, 1980; Holt \& Saunders, 1983, 1984) means that it is now feasible to obtain recombinants of genetically diverse fungi. From such hybrids strain improvement procedures could lead to the production of the new antibiotics at a commercially acceptable level. If hybridization experiments are to be carried out in a search for new metabolites then an initial problem is to decide which strains should be recombined (Holt \& Saunders, 1983). This paper describes one approach, whereby mixed cultures are screened for activity not shown by either of the component strains. Isolates of $\boldsymbol{A}$. nidulans and $\boldsymbol{A}$. flavus were chosen as a model system since strains of each genetically labelled on eight linkage groups are available for possible detailed study of interspecific recombination.

\section{METHODS}

Strains and culture conditions. The genotype and origins of the strains of Aspergillus nidulans (GH79) and $\boldsymbol{A}$. flavus (CMI 91019B) employed are given in Table 1. Aspergillus nidulans carried a number of genetical markers including the mutation npeA which results in impaired penicillin production (Holt et al., 1976). A prototrophic strain of $A$. flavus was used.

Culture conditions, media and levels of nutritional supplements for routine growth were based on those of Pontecorvo et al. (1953) as adapted by Clutterbuck (1974). Submerged liquid cultures for antibiotic production were shaken at $25^{\circ} \mathrm{C}$ on an orbital shaker $(200$ r.p.m., $5 \mathrm{~cm}$ throw) in $100 \mathrm{ml}$ polypropylene flasks containing $20 \mathrm{ml}$ of the antibiotic fermentation medium described by Holt \& Macdonald (1968) but lacking the penicillin side chain precursor, phenylacetic acid. Except where stated, inoculations were made with $1 \mathrm{ml}$ of spore

† Present address: National Institute for Biological Standards and Control, Holly Hill, Hampstead, London NW3, UK.

$\ddagger$ Present address: Genetic Engineering Research Department, Bristol Myers Co., PO Box 657, Syracuse, New York 13201, USA. 
Table 1. Details of micro-organisms used

$\quad$ Organism
Aspergillus flavus
Aspergillus nidulans
Bacillus subtilis
Escherichia coli
Proteus vulgaris
Pseudomonas aeruginosa
Serratia marcescens
Staphylococcus aureus

Organism

Aspergillus flavus

Bacillus subtilis

Escherichia coli

Proteus vulgaris

Serratia marcescens

Staphylococcus aureus

$\left.\begin{array}{l}\multicolumn{1}{c}{\text { Origin* }} \\ \text { CMI 91019B } \\ \text { PCL (GH79) } \\ \text { NCTC } 8236 \\ \text { NCTC } 86 \\ \text { NCTC } 4635 \\ \text { NCIB } 8295 \\ \text { NCTC } 8706 \\ \text { ATCC } 27735\end{array}\right\}$

\author{
Genotype \\ Prototrophic \\ yA pyroA cnxA npeA $\dagger$ \\ Prototrophic strains of test \\ organisms used for the \\ antibiotic bioassay
}

* CMI, Commonwealth Mycological Institute; PCL, Polytechnic of Central London; NCTC, National Collection of Type Cultures, UK; NCIB, National Collection of Industrial Bacteria; ATCC, American Type Culture Collection.

† Gene symbols: $y A$, yellow conidia; pyro $A$, auxotrophic requirement for pyridoxin; $c n x A$, requirement for reduced nitrogen; npeA, impaired in penicillin production.

suspensions containing approximately $10^{7}$ spores $\mathrm{ml}^{-1}$. Bioassays of culture filtrates were performed according to the procedures outlined by Brownlee $e t$ al. (1948) and included $\beta$-lactamase (Sigma) in the agar at a final concentration of 80 units $\mathrm{ml}^{-1}$ in order to hydrolyse any $\beta$-lactam antibiotics produced.

Purification procedure. Compounds VI and VII were extracted from the fermentation liquor (pH 7.0) into ethyl acetate. The solvent extract was concentrated in vacuo and applied to a column of silica gel. The column was developed with ethyl acetate to elute compound VI, then the solvent was changed to ethyl acetate/methanol $(75: 25, \mathrm{v} / \mathrm{v})$ to elute compound VII.

Those fractions containing compound VI were pooled and the solvent removed in vacuo. The preparation was dissolved in $0 \cdot 1 \mathrm{M}-\mathrm{Na}_{2} \mathrm{CO}_{3}$, extracted with benzene and the solvent layers discarded. The aqueous phase was acidified with $\mathrm{HCl}$ and extracted with ethyl acetate. Removal of the solvent in vacuo yielded compound VI as a yellow crystalline product which was homogeneous under TLC. A crude melting point of $142-145^{\circ} \mathrm{C}$ was recorded for the product, which was obtained in too low a yield to permit recrystallization.

After fractionation over silica gel, the fractions containing compound VII were combined and the solvent removed in vacuo. The preparation was then dissolved in methanol and applied to a column of Sephadex LH-20 developed with the same solvent. Those fractions containing compound VII were pooled and removal of the solvent in vacuo yielded this antibiotic as a yellow crystalline product, crude melting point $93-98^{\circ} \mathrm{C}$, which was homogeneous on TLC but could not be recrystallized due to the low yield.

Gas-liquid chromatography. This was carried out on a Perkin Elmer F11 chromatograph equipped with an FID detector and a $1 \mathrm{~m} \mathrm{OV} 1$ column. The oven temperature was initially $200^{\circ} \mathrm{C}$ and was programmed to increase at $5^{\circ} \mathrm{C} \mathrm{m^{-1 }}$. Injection temperature was approximately $280^{\circ} \mathrm{C}$, the carrier gas was nitrogen at $20 \mathrm{ml} \mathrm{min}^{-1}$, the range was 10 , and the attenuation was 16 with an injected sample of $2 \mu l$.

Trimethylsilyl derivatives of compound VI and neohydroxyaspergillic acid were prepared by reacting $1.5 \mathrm{mg}$ of each with $0.5 \mathrm{ml}$ Regisil RC-2 (Phase Separations Ltd, Deeside Industrial Estate, Queensferry, Clwyd CH5 2LR, UK) at $120^{\circ} \mathrm{C}$ for $2 \mathrm{~h}$.

\section{RESULTS}

A strain of $A$. nidulans (GH79) impaired in penicillin production was chosen as one of the component cultures, in order to facilitate recognition of antibiotic activity other than $\beta$-lactams even when tested against $\beta$-lactam sensitive bacteria. It was grown in mixed culture with a prototrophic strain of $A$. flavus (CMI 91019B). This combination yielded an activity against Escherichia coli and Staphylococcus aureus which was not present when either fungus was grown alone. Peak titres were observed after $6 \mathrm{~d}$ growth. Examination of the culture filtrate revealed the presence of two antibiotics designated VI and VII (V and other antibiotics produced by strains of $A$. nidulans have been described elsewhere : Cole, 1980; Cole et al., 1976; Middleton et al., 1979; Perry et al., 1982a, b).

Chemical properties. Compounds VI and VII both formed red-coloured complexes with ferric chloride and green-coloured cupric salts, which is consistent with their possessing hydroxamic acid structures. They were also resistant to acidic and alkaline hydrolysis, suggesting that the hydroxamic acid group is part of a heterocyclic system and not of the simple aliphatic type. 


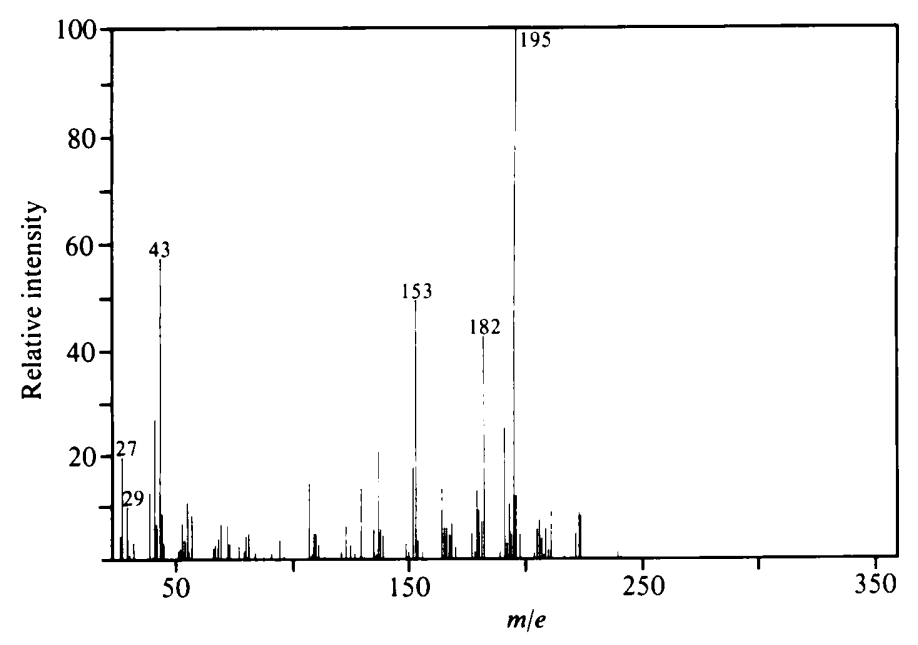

Fig. 1. Mass spectrum of compound VI.

Antibiotic activity. No minimum inhibitory concentration (MIC) values were obtained for compound VII, although this antibiotic has been observed to inhibit the growth of Bacillus subtilis, $E$. coli and $S$. aureus. MIC values for compound VI were obtained using an agar plate diffusion assay in which $1 \mathrm{~cm}$ diameter discs of filter paper (Whatman no. 1) were impregnated with compound VI. The agar used was diagnostic sensitivity test agar (Oxoid) which had an iron content of $1.2 \mu \mathrm{g} \mathrm{ml}^{-1}$. The MIC values were $\left(\mu \mathrm{g} \mathrm{ml}^{-1}\right)$ : B. subtilis, 26; E. coli, 14; Pseudomonas aeruginosa, 70; Proteus vulgaris, 98; Staphylococcus aureus, 30; Serratia marcescens, > 100.

Mass spectroscopy. Mass spectrometry of compound VI was done by the Physico-Chemical Measurements Unit, Harwell, Berks., UK. The molecular ion was at $m / e$ 240.1481 and the suggested formula was $\mathrm{C}_{12} \mathrm{H}_{20} \mathrm{O}_{3} \mathrm{~N}_{2}$ (see Fig. 1).

Infrared spectroscopy. Compound $\mathrm{VI}$ in a $\mathrm{KBr}$ disc exhibited the following absorption maxima: 2945, 2920,2880,1580,1530,1410,1230,1160,1120,1040 and $1000 \mathrm{~cm}^{-1}$.

Ultraviolet spectroscopy. In ethanol, the UV spectra of compounds VI and VII were very similar, exhibiting absorptions centred at 212,232 and $333 \mathrm{~nm}$.

The chemical and spectroscopic analysis of compound VI strongly indicated that it was a hydroxylated member of the aspergillic acid group of mycotoxins known to be produced by several Aspergillus species (Steyn, 1980). Compound VI was therefore compared with an authentic sample of neohydroxyaspergillic acid kindly supplied by $\mathrm{Dr}$ L. Delcambe, International Centre of Information on Antibiotics, Boulevard de la Constitution 32, B-4020 Liège, Belgium. Although the two components were indistinguishable by TLC, they were well resolved by GLC. The tetramethylsilyl derivative of component VI had a retention time of 6.4 min compared with $5.5 \mathrm{~min}$ for TMS-neohydroxyaspergillic acid. The mass spectrum of component VI differed significantly from that reported for $\beta$-hydroxyneoaspergillic acid (Mabayashi et al., 1978). According to Steyn (1980), therefore, three structures were likely (Fig. 2). The mass spectrum of compound VI showed prominent peaks at $m / e 195,182,153,43,29$ and 27 which may arise thus: 195 , loss of $\mathrm{O}$ from $\mathrm{M}^{+}(240)$ followed by loss of $\mathrm{C}_{2} \mathrm{H}_{5} ; 182$, loss of $\mathrm{O}$ from $\mathrm{M}^{+}$followed by loss of $\mathrm{C}_{3} \mathrm{H}_{6}$ by McLafferty rearrangement; 153, loss of $\mathrm{C}_{2} \mathrm{H}_{5}$ from 182 or $\mathrm{C}_{3} \mathrm{H}_{6}$ from 195 by McLafferty rearrangement; $43, \mathrm{C}_{3} \mathrm{H}_{7}{ }^{+}$ion; $29, \mathrm{C}_{2} \mathrm{H}_{5}{ }^{+}$ion; $27, \mathrm{C}_{2} \mathrm{H}_{3}{ }^{+}$ion.

Structure (I) was discounted as the loss of $\mathrm{C}_{2} \mathrm{H}_{5}$ from $\mathrm{m} / \mathrm{e} 182$ to give $\mathrm{m} / \mathrm{e} 153$ was not possible. As (III) was thought unlikely to give rise to $\mathrm{C}_{3} \mathrm{H}_{7}$, compound VI was probably hydroxyaspergillic acid (II). In addition, the crude melting point obtained for compound VI $\left(142-145^{\circ} \mathrm{C}\right)$ was consistent with that reported for hydroxyaspergillic acid $\left(148-150^{\circ} \mathrm{C}\right)$ (Steyn, 1980). 
<smiles>CC(C)C1=NCC(C(O)C(C)C)C1O</smiles>

I Neohydroxyaspergillic acid<smiles>CCC(C)(C)C1CN=C(C(C)C)C(O)N1O</smiles>

II Hydroxyaspergillic acid<smiles>CCC(C)C1NCC(C(C)(O)C(C)C)N1O</smiles>

III 1-Hydroxy-3-sec-butyl-6-(1-hydroxy-1-methyl-propyl)-pyrazin-2-one

Fig. 2. Structural formulae of hydroxyaspergillic acids. The compounds are shown in the hydroxamic acid tautomeric forms which are believed to be responsible for their antibiotic properties.

The production of aspergillic acids by fungi in liquid culture is known to be both strain- and medium-specific (Middleton et al., 1979) and to be influenced by the size of the inoculum. Thus the detection of antibiotic activity could have resulted from an increase in the innate production of some hydroxamic acid by one strain from a level below, to one above, the sensitivity of the bioassay system as a result of the physiological conditions of the culture rather than a de novo biosynthetic reaction between the two species. To test this possibility two further experiments were performed. (a) Pure cultures of $A$. nidulans GH79 and A. flavus CMI 91019B were tested for activity against $E$. coli after $6 \mathrm{~d}$ submerged shaken culture in $20 \mathrm{ml}$ fermentation medium inoculated with different amounts of fungal conidia (from $10^{2}$ to $\left.10^{9}\right)$. (b) Filtrates $(100 \mathrm{ml})$ of pure cultures were extracted with $100 \mathrm{ml}$ chloroform, and the extract was evaporated to dryness in vacuo, redissolved in $10 \mathrm{ml}$ water and tested in bioassay against $E$. coli. This procedure would have concentrated tenfold any aspergillic acids present. Under each condition neither fungal strain alone produced an antibiotic active against $E$. coli.

\section{DISCUSSION}

A mixed culture of $A$. nidulans GH79 and A. flavus CMI 91019B produced two antibiotics, VI and VII, which were not elaborated by pure cultures of either strain. Chemical and spectroscopic analysis of compound VI strongly suggested that this antibiotic was hydroxyaspergillic acid. Unfortunately compound VII was not produced in sufficient quantity for a detailed analysis to be feasible. However, it gave a typical hydroxamate reaction with both ferric and cupric chloride and had a UV spectrum similar to that of compound VI. We therefore believe that compound VII is also probably a member of the aspergillic acid group of metabolites. Anke (1977) has shown that the activity of hydroxamic acid antibiotics is sensitive to environmental iron levels. However, compounds VI and VII were both produced in a complex fermentation medium with an unknown iron content.

Although aspergillic acid and its derivatives are known to be produced by certain strains of $A$. flavus (Steyn, 1980) they have not been isolated from $A$. nidulans. The mechanisms by which the biosynthetic pathways of $A$. nidulans GH79 and $A$. flavus CMI 91019B interact to produce compounds VI and VII are unknown but could include: (1) the biochemical transformation of metabolites of one species by the other; (2) deregulation or suppression of biosynthetic pathways; and (3) complex cosynthetic interactions. 
The results presented here demonstrate the feasibility of obtaining detectable levels of interesting metabolites from mixed cultures, which could thus provide a new source of biologically useful molecules. Studies on the generation of such metabolic novelty, by hybridization using protoplast fusion of genetically marked strains of Aspergillus species, are underway.

\section{REFERENCES}

ANKE, H. (1977). Metabolic products of microorganisms. 163. Desferritriacetylfusigen, an antibiotic from Aspergillus deflectus. Journal of Antibiotics 30, 125-128.

Brownlee, K. A., Loraine, P. K. \& Stephens, J. (1948). The biological assay of penicillin by a modified plate method. Journal of General Microbiology 3, 347-352.

ClutterbuCK, A. J. (1974). Aspergillus nidulans. In Handbook of Genetics, vol. 1, pp. 447-510. Edited by R. C. King. New York \& London: Plenum Press.

Cole, D. S. (1980). Products of Aspergillus nidulans. Aspergillus Newsletter 14, 24-26.

Cole, D. S., Edwards, G. F. St L., Holt, G. \& Macdonald, K. D. (1976). Antibiotic production by wild type and mutant strains of Aspergillus nidulans. In Fifth International Fermentation Symposium, p. 193. Edited by H. Dellweg. Berlin: Verlag Versuchsund Lehranstalt für Spiritusfabrikation und Fermentationstechnologie.

FERENCZY, L. (1979). Fusion of protoplasts of autotrophic fungal mutants: diversity in the genetic background of nutritional complementation. In Advances in Protoplast Research, pp. 55-62. Edited by L. Ferenczy \& G. L. Farkas. Oxford: Pergamon Press.

Holt, G. \& Macdonald, K. D. (1968). Penicillin production and its mode of inheritance in Aspergillus nidulans. Antonie van Leeuwenhoek 34, 409-416.

HolT, G. \& SAUNDERS, G. (1983). Genetics and breeding of antibiotic-producing microorganisms. Rivista di biologia 76, 183-193.

Holt, G. \& Saunders, G. (1984). Genetic modification of industrial microorganisms. In Comprehensive Biotechnology, vol. 1. Edited by M. Moo-Young. Oxford: Pergamon Press (in the Press).
Holt, G., Edwards, G. R. St L. \& Macdonald, K. D. (1976). The genetics of mutants impaired in the biosynthesis of penicillin. In Proceedings of the Second International Symposium on the Genetics of Industrial Microorganisms, pp. 199-211. Edited by K. D. Macdonald. London; Academic Press.

Hopwood, D. A. \& Chater, K. F. (1980). Fresh approaches to antibiotic production. Philosophical Transactions of the Royal Society B290, 313-328.

Mabayashi, Y., Sumita, M., FukUshine, K. \& YAMAZAKI, M. (1978). Isolation and structure of a red pigment from Aspergillus ochraceus. Chemical and Pharmaceutical Bulletin 26, 1320-1322.

Makins, J. F., Holt, G. \& Macdonald, K. D. (1980). Cosynthesis of penicillin following treatment of mutants of Aspergillus nidulans impaired in antibiotic production with lytic enzymes. Journal of General Microbiology 119, 397-404.

Middleton, A. J., Cole, D. S. \& Macdonald, K. D. (1979). A hydroxamic acid from Aspergillus nidulans with antibiotic activity against Proteus species. Journal of Antibiotics 31, 1110-1115.

Perry, M. J., Adlard, M. W. \& Holt, G. (1982a). The isolation of a fungal metabolite which exhibits synergy with sterigmatocystin. Journal of Applied Bacteriology 52, 83-89.

Perry, M. J., Adlard, M. W. \& Holt, G. (1982b). The effect of the synergy between sterigmatocystin and a fungal metabolite on Bacillus subtilis. Journal of Applied Bacteriology 52, 91-96.

Pontecorvo, G., Roper, J. A., Hemmons, L. M., Macdonald, K. D. \& Bufton, A. W. J. (1953). The genetics of Aspergillus nidulans. Advances in Genetics $5,141-238$.

STEYN, P. S. (1980). The Biosynthesis of Mycotoxins. London: Academic Press. 